\title{
Path structures on manifolds
}

\author{
Jürgen Ehlers and Egon Köhler \\ Max-Planck-Institut für Physik und Astrophysik, München, F. R. Germany \\ (Received 7 February 1977)
}

We study collections of paths-i.e., unparametrized curves - on a manifold such that through every point and every direction at that point there passes exactly one path. Among such path structures we characterize, analytically and in terms of symmetries, those which consist of geodesics of a linear connection. Examples of nongeodesic path structures are given, and some of the results are interpreted physically.

An axiomatic approach to the spacetime structure of general relativity can be based on the following concepts: a four-dimensional manifold $M$, a Lorentzian conformal structure $C$ on $M$, and a projective structure $P$ on $M$ compatible with $C .{ }^{1}$ The points of $M$ are considered as mathematical images of events in physical spacetime, the conformal structure represents the causal structure as indicated in particular by the propagation of light, and the projective structure represents inertia which, according to Einstein, is identical with gravity, and which manifests itself particularly simply in free fall motions of test particles.

Causal structures per se have been investigated in detail by several authors. ${ }^{2}$ Similar studies concerning inertial structures which might lead to a deeper understanding of Einstein's law of inertia seem to be lacking.

The world line of a freely falling neutral test particle is uniquely determined by one of its event and its direction at that event. One is, therefore, led to consider collections of paths-unparametrized curves (see Sec. 1)- such that given a point $p$ and $a$ direction $\xi$ at $p$, there is exactly one path going through $p$ in the direction $\xi$. The standard example of such a path structure consists of the paths represented by geodesics of a linear connection. The question thus arises: How can one characterize, analytically and geometrically, the geodesic path structures among the general ones? One would like particularly to have characterizations which can be interpreted physically.

In this paper we shall give one convenient analytic characterization of geodesic path structures (Theorem 1 in Sec. 3) and two geometrical ones (Theorems 2 and 3 in Sec. 4). According to Theorem 3, Einstein's law of inertia (using geodesics to represent free fall world lines) is equivalent to the statement: The set of free fall world lines is a path structure with the following property (of "local isotropy"): The collection of all path elements passing through an event $p$ is invariant under a group of local diffeomorphisms which acts transitively on the set of all bases of the direction space (see Sec. 1) at $p$. (A path element is an equivalence class of paths which have a second-order contact at $p$; intuitively it is a second-order infinitesimal piece of a path.) So, roughly speaking, the set of all free fall world lines has the highest possible degree of local isotropy which a path structure can possess.

In Secs. 1 and 2 we develop the appropriate concepts to deal with path structures. Sections 3 and 4 contain the main results indicated above, and Sec. 5 gives some examples of nongeodesic path structures.

\section{DIRECTIONS AND PATHS}

Let $M$ be a connected, paracompact Hausdorff manifold of class $C^{k}(k \geqslant 3)$ and dimension $n \geqslant 2$. By $T_{p} M$ we denote its tangent space at $p \in M$, and by $\pi_{T}: T M \rightarrow M$ its tangent bundle. Every $C^{k}$ curve $\gamma: I \rightarrow M(I \subset \mathbb{R})$ has a (canonical) lift $\gamma^{2}: I \rightarrow T M$, which we call a special curve in $T M$. The vectors $\dot{\gamma}^{T}(s) \in T_{\gamma} T_{(s)} T M$ are the tangents to $\gamma^{T}$. We call a vector $X \in T^{2} M$ a special vector, if it can be obtained as a tangent vector of a special curve in $T M$.

If $\pi_{T^{*}}$ is the differential of $\pi_{T}$ and $\Pi_{T}$ the bundle projection $\Pi_{T}: T^{2} M \rightarrow T M$, then special vectors are characterized by $\pi_{T^{*}} X=\Pi_{T} X$. A special $C^{1}$ vector field $X$ : $T M \rightarrow T^{2} M$ is called a differential equation of second order. Its integral curves are special curves in $T M{ }^{3}$

In the sequel we shall specialize the concept of a "curve in $M$ " by requiring: If $\gamma\left(s_{1}\right)=\gamma\left(s_{2}\right)$ and $\dot{\gamma}\left(s_{1}\right)=\gamma\left(s_{2}\right)$, then there exist open intervals $I_{1}, I_{2}$ and a smooth, invertible map $\mu: I_{1} \rightarrow I_{2}$ such that $\mu\left(s_{1}\right)=s_{2}$ and $\gamma \mid I_{1}$ $=(\gamma \circ \mu) \mid I_{1}$. (This excludes self-tangency.)

In many physical applications the parametrization of a curve is arbitrary or not specified a priori. Therefore, we need the concept of a path $\Gamma$ as a "curve without parameter" or, more rigorously, as an equivalence class of curves with nowhere vanishing tangents which differ only by a parameter transformation. ${ }^{4}$ Each curve $\gamma$ defines a path $\gamma \cdot \gamma$ is a representative of $\Gamma, \gamma \in \Gamma$, if $\chi=\Gamma$.

We call an equivalence class $\xi$ of vectors $X, Y, \cdots$ $\in T_{p} M$ which are proportional, $X=\lambda Y(\lambda \neq 0)$, a direction and write $\xi=X=Y=\cdots$. The directions generated by all vectors of $T_{p} \vec{M} \backslash\{0\}$ form the direction space $D_{p} M$ which is isomorphic to $\mathrm{IP}^{n-1}$. A path $\Gamma=\mathcal{\chi}$ has a (unique) direction $\dot{\gamma}(s)$ at each of its points $\gamma(s)$.

The collection of all $D_{p} M$ over $M$ forms another fiber bundle $D M$ with projection $\pi_{D}$ and compact fibers $D_{p} M$. For every curve $\gamma$ in $M$ with $\dot{\gamma} \neq 0$ there exists a canonical lift $\dot{\gamma}$ into $D M$, defined by

$$
\dot{\dot{\gamma}}: I \rightarrow D M: s \rightarrow \dot{\gamma}(s) .
$$

We call such curves special curves in $D M$.

Equivalent curves $\gamma \sim \mu$ (i.e., curves which represent the same path $\Gamma$ ) have equivalent lifts, $\dot{\gamma} \sim \dot{\mu}$. Therefore, a path $\Gamma=\underset{\sim}{\gamma}$ in $M$ generates a unique path $\underline{\bar{\gamma}}$ in $D M$ which 
we denote by $\dot{\Gamma}$ (the lift of $\Gamma$ to $D M$ ). As before a path in $D M$ is said to be special, if it is the lift of a path in $M$.

$D M$ is a $C^{k-1}$ manifold, so we can construct the bundles $T D M$ and $D D M=D^{2} M$ with the respective projections $\hat{\Pi}$ and $\Pi_{D}$. Besides $\Pi_{D}$ there is another natural map from $D^{2} M$ to $D M$ which is analogous to $\pi_{T^{*}}: T^{2} M \rightarrow T M$. It is obtained as follows. Define

$$
\begin{aligned}
& R_{1}: T M \rightarrow D M: X \rightarrow \underline{X}=\xi, \\
& R_{2}: T D M \rightarrow D^{2} M: \xi \rightarrow \underline{\xi}=\Xi,
\end{aligned}
$$

and consider the diagram

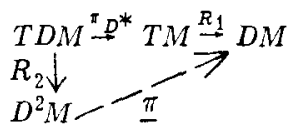

Since any two elements $\xi_{1}, \xi_{2} \in R_{2}^{-1} \Xi, \Xi \in D^{2} M$, have the same image under $R_{1} \circ \pi_{D^{*}}$, there exists a map $\pi$ $=R_{1}{ }^{\circ} \pi_{D^{*}} \circ R_{2}^{1}$ as indicated. In close analogy to the definition of special vectors in $T^{2} M$ we define: $\Xi \in D^{2} M$ is a special direction, if

$$
\Pi_{D}(\Xi)=\underline{\pi}(\Xi) \text {. }
$$

Special paths in $D M$ have everywhere special directions directions. Moreover, every special direction field of class $C^{1}$

$$
\Xi: D M \rightarrow D^{2} M: \xi \rightarrow \Xi_{\xi},
$$

determines unique maximal special integral paths in $D M$ and in the original manifold $M$.

\section{PATH STRUCTURES}

Definition: A path structure (PS) $P$ on $M$ is a set of paths in $M$ such that for every point $p \in M$ and every direction $\xi_{p} \in D_{p} M$ there exists exactly one maximal path $\Gamma \in P$ which contains $p$ and has the direction $\xi_{p}$ at $p$.

The definition implies that through every point $\xi \in D M$ runs exactly one lifted path $\dot{\Gamma}$ of a path $\Gamma \in P$. Moreover, due to the restriction imposed on "curves in $M$, " $\dot{\Gamma}$ has no self intersections whence we have the following lemma.

Lemma: A path structure $P$ on $M$ defines a special direction field (section of $D^{2} M$ ),

$$
\Xi: D M \rightarrow D^{2} M: \xi \rightarrow \Xi \xi
$$

such that for every path $\Gamma \in P$ and every point $\xi$ on $\dot{\Gamma}$

$$
\ddot{\Gamma}_{\xi}=\Xi_{\xi} \text {. }
$$

This lemma enables us to define the differentiability class of a PS.

Definition: A path structure $P$ is of class $C^{s}$ if the corresponding section $\Xi$ is of class $C^{s}$. We always demand $s \geqslant 1$.

Any special $C^{1}$-direction field over $D M$ determines special maximal integral paths in $D M$ whose projections to $M$ satisfy the definitions given above which proves the following lemma.

Lemma: A special direction field of class $C^{s}, s \geqslant 1$, defines a $C^{s}$-path structure on $M$.
According to the preceding lemmas there is a one-toone correspondence between smooth PS's and special direction fields. For the local analytical investigation of PS's special direction fields play a role analogous to that of second order differential equations for systems of curves.

For the futher analysis of PS's we describe direction fields in local coordinates of $D^{2} M$ introduced as follows. Choose any local coordinate system $\left(x^{a}\right)$ for $U \subset M$ and use standard coordinates $\left(x^{a}, y^{b}\right)$ in $T U$ such that a vector $X \in T U$ is given by

$$
X=y^{a} \frac{\partial}{\partial x^{a}} \cdot
$$

In $T^{2} U$ we also define standard coordinates $\left(x^{a}, y^{b}, u^{\mathrm{c}}\right.$, $\left.w^{d}\right)$ by

$$
\mathbf{X}=u^{a} \frac{\partial}{\partial x^{a}}+w^{b} \frac{\partial}{\partial y^{b}} \in T^{2} U
$$

Special vector fields over $T U$ are characterized in these coordinates by $y^{b}=u^{b}$, so that the most general special vector field is given by

$$
\mathrm{X}=y^{a} \frac{\partial}{\partial x^{a}}+f^{b}\left(x^{c}, y^{d}\right) \frac{\partial}{\partial y^{b}}
$$

with smooth functions $f^{b}$.

In $D U$ we introduce coordinates $\left(x^{a}, \xi^{\beta}\right) \beta=1, \ldots, n-1$, based on $\left(x^{a}, y^{b}\right)$, by $\xi^{\beta}:=y^{\beta} / y^{n}$ (for $y^{n} \neq 0$ ). If $y^{n}=0$ we use equation $\xi_{b}^{\beta}:=y^{\beta} / y^{b}(\beta=1, \ldots, b-1, b+1, \ldots, n)$ with $y^{b} \neq 0 。 \pi_{D}^{-1}(U)$ is covered by these $n$ coordinate neighborhoods. Unless more than one of these patches is necessary we use the $n$th one with $\left(x^{a}, \xi_{n}^{\beta}\right)=\left(x^{a}, \xi^{\beta}\right)$.

This procedure can be repeated to obtain coordinates in $D^{2} U$. In $T D U$ there are standard coordinates $\left(x^{a}, \xi^{\beta}\right.$, $\left.u^{c}, \eta^{6}\right)$ such that

$$
\xi=u^{a} \frac{\partial}{\partial x^{a}}+\eta^{\beta} \frac{\partial}{\partial \xi^{\beta}} \in T D U .
$$

To get coordinates in $D^{2} U$ the $2 n-1$ quantities $\left(u^{a}, \eta^{\beta}\right)$ can be divided by one of its nonzero members. However, if $\Xi \in D^{2} U$ is a special direction (and only those are of interest to us), then it turns out that there exists always one nonzero component of the $\left(u^{a}\right)$. For special directions only $n$ coordinate patches are required, defined by

$$
\left(x^{a}, \xi_{b}^{\beta}, \mu_{b}^{\gamma}, v_{b}^{\delta}\right)=\left(x^{a}, \frac{y^{8}}{y^{b}}, \frac{u^{\gamma}}{y^{b}}, \frac{\eta^{\delta}}{y^{b}}\right), \quad b=1, \ldots, n .
$$

$b=n$ will be omitted as before.

Furthermore, a special direction field is characterized by $\xi^{\beta}=\mu^{\beta}$ whence such a field is completely described by $n-1$ functions of $2 n-1$ variables

$$
v^{\gamma}=g^{\gamma}\left(x^{a}, \xi^{\beta}\right) \text {. }
$$

Note that prescribing the functions $g^{\gamma}$ in one coordinate patch of $D^{2} M$ (the $n$th one in this case) determines all the other $v_{b}^{\gamma}\left(x^{a}, \xi_{b}^{\beta}\right)$.

\section{GEODESIC PATH STRUCTURES}

The most important PS's are given by the geodesics of a linear connection $\Gamma$ on $M$. (Such connections always exist since $M$ is paracompact.) In local coordinates the 
geodesics are given as the solutions of the equations

$$
\ddot{x}^{a}=-\Gamma_{b a}^{a} \dot{x}^{b} \dot{x}^{c} .
$$

These geodesics define a path structure $P_{\Gamma}$ and a corresponding direction field $\Xi_{\Gamma}$. In terms of the coordinates defined above $\Xi_{\Gamma}$ is given by

$$
\Xi_{\Gamma}=\left(x^{a}, \xi^{\beta}, \xi^{\gamma}, v^{6}\right)=\left(x^{a}, \frac{x^{\beta}}{x^{n}}, \frac{\dot{x}^{\gamma}}{\dot{x}^{n}}, \frac{\ddot{x}^{6} \dot{x}^{n}-\ddot{x}^{n} \dot{x}^{5}}{\left(\dot{x}^{n}\right)^{3}}\right)
$$

or, using (1) and putting $\xi^{\mathrm{n}}:=1$,

$$
v^{6}=2 \Gamma_{(c d}^{[n} \delta_{e)}^{6]} \xi^{c} \xi^{d} \xi^{e} \text {. }
$$

Thus $v^{6}$ is a polynomial in $\xi^{\alpha}$ of maximal degree 3 . Clearly a projective change of the connection or a change of its torsion does not affect the functions $v^{6} ; \Xi_{\Gamma}$ is determined by the projective PS implied by $\Gamma$.

Equation (3) shows further that $v^{6}$ does not contain all monomials of degree 3 (except for $n=2$ ) and that the coefficients of the other terms are not independent for different indices $\delta$. Trying to construct a direction field given by $v^{6}$ 's which are polynomials, but cannot be written in the form (3), one finds that there exists a discontinuity in at least one of the other coordinate patches. Thus such a direction field is not even $C^{0}$. We illustrate this by a simple

Example: $n=2$ : Consider both coordinate patches,

$$
\left(x^{a}, \xi_{2}^{1}, v_{2}^{1}\right)=\left(x^{a}, \xi, v\right) \text { and }\left(x^{a}, \xi_{1}^{2}, v_{1}^{2}\right)=\left(x^{a}, \bar{\xi}, \bar{v}\right) \text {. }
$$

Take

$$
v=\xi^{b}, p>3 \text {. }
$$

From (2) we get $\bar{\xi}=\xi^{-1}$ and

$$
\bar{v}=-\bar{\xi}^{3-p}
$$

which is not continuous for $\bar{\xi}=0$.

Similarly it can be shown for $n>2$ that if a $C^{1}$ path structure is given by polynomials $v^{5}\left(\xi^{\alpha}\right)$, then these polynomials can be written in the form (3). Because of the 1-1 correspondence between polynomials (3) and geodesic PS's we can reformulate this result as our first characterization of geodesic path structures:

Theorem 1: For a given special direction field $\Xi$ of class $C^{1}$ there exists a linear connection $\Gamma$ whose geodesics generate the PS of $\Xi$, if and only if the coordinates $v^{\alpha}$ of $\Xi$ are polynomials in $\xi^{\beta}$. These polynomials are necessarily of the form (3), and $\Gamma$ is determined by $\Xi$ up to torsion and projective changes.

\section{SYMMETRIES OF PATH STRUCTURES}

According to a famous theorem of Helmholtz (1868) Riemannian spaces can be characterized among the more general metric spaces as being infinitesimally isotropic. ${ }^{5}$ Since this is an intuitively appealing characterization (expressing free mobility of small rigid bodies) the purpose of this section is to characterize geodesic path structures in a similar way in terms of symmetries. In this section we shall show how this can be done.

We first define (finite) symmetries of PS's. Let $\phi$ : $M \rightarrow M$ be a diffeomorphism of class $C^{h} . \phi$ induces a mapping of the paths $\Gamma \in P$ onto a set of paths $\Gamma^{\prime}$, which again form a PS, $p^{\Phi}$. Let us reformulate this operation in terms of special direction fields. The differential of $\phi$,

$$
\phi_{*}: T M \rightarrow T M,
$$

induces a diffeomorphism

$$
\Phi_{*}:=\Phi_{*}: D M \rightarrow D M \text { via } \underline{\phi}_{*}(\xi)=\underline{\left(\phi_{*} X\right)}
$$

for $X \in \xi$. In the same way the differential

$$
\underline{\left(\phi_{*}\right)_{*}: T D M \rightarrow T D M}
$$

induces the mapping

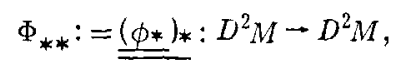

for which the following is true:

Lemma: A diffeomorphism $\phi: M \rightarrow M$ of class $C^{k}$, $k \geqslant 3$, induces diffeomorphisms

$$
\Phi_{*}: D M \rightarrow D M \text { and } \Phi_{* *}: D^{2} M \rightarrow D^{2} M
$$

such that for every special direction field $\Xi: D M \rightarrow D^{2} M$

$$
\Xi^{\oplus}:=\Phi_{* *}^{\circ} \Xi \circ \Phi_{*}^{-1}
$$

is again a special direction field.

If $\Xi$ corresponds to $P$, then $\Xi^{\Phi}$ corresponds to $P^{\Phi}$.

Definition: $\phi$ is called a symmetry of the path structure $P$ if $p^{\Phi}=P$ or, equivalently, if the corresponding direction field $\Xi$ is invariant under $\phi$,

$$
\Xi^{\Phi}=\Xi \text { or } \Phi_{* *} \Xi_{\xi}=\Xi_{\Phi * \xi} \text {. }
$$

A local symmetry of $P$ is a local diffeomorphism $\phi$ : $U \rightarrow V$ of $M$ which maps the restrictions of $P$-paths to $U$ into restrictions of $P$-paths to $V$.

In order to consider isotropy of path structures, we denote by $p_{p}$ the subset of $p$ whose members pass through $p$, and formulate the following definition.

Definition: A $P$-rotation about $p$ is a local diffeomorphism $\phi: U \rightarrow V$ of $M$ with fixed point $p$ which maps the restrictions of elements of $P$, to $U$ into restrictions of elements of $P_{p}$ to $V$.

The set of all symmetries of a path structure is a group $G(P)$, whereas the local symmetries of $p$ and the $p$-rotations (for some point $p$ ) form pseudogroups. PS's determined by projectively flat linear connections have symmetry groups (or pseudogroups) acting transitively not only on $D_{\mathrm{p}} M$ but even on the set of projective bases of $D_{p} M$.

Before establishing a converse of the last assertion we introduce a weakened, infinitesimal analog of the concept of a $P$-rotation, guided by the analogous Helmholtz theorem. For this purpose we observe that if $p$ is a fixed point of a local diffeomorphism $\phi$ of $M$, then $\Phi_{*}$ maps $D_{p} M$ onto itself projectively and $\Phi_{* *}$ maps $\Pi_{D}^{-1}$ $\left(D_{p} M\right)$ into itself, so that it is meaningful to restrict the second Eq. (4) to $D_{p} M$. Accordingly, we formulate the following definition.

Definition: An approximate P-symmetry ${ }^{6}$ (APS) at $p$ is the restriction to $\Pi_{D}^{-1}\left(D_{p} M\right)$ of a local diffeomorphism $\Phi_{* *}$ of $D^{2} M$, induced by a local diffeomorphism $\phi$ of $M$ 
which leaves $p$ unchanged and satisfies the following condition:

$$
\xi \in D_{p} M \Rightarrow \Phi_{* *} \Xi_{\xi}=\Xi_{\Phi_{*} \xi}
$$

An $A P S$ at $p$, say $\Psi$, uniquely determines a projective isomorphism $\tilde{\Psi}: D_{p} M \rightarrow D_{p} M$ since $\Psi$ maps fibres into fibres. The set of all $A P_{S}$ 's at $p$ is a Lie group $\widetilde{G}_{p}(p)$, and the set of the associated maps $\tilde{\Psi}$ is a Lie subgroup $\widetilde{G}_{p}(P)$ of the full projective group $P G\left(D_{p} M\right)$ of $D_{p} M$. In fact, the map sending $\Psi$ into $\tilde{\Psi}$ is a homomorphism,

$$
G_{p}(p) \rightarrow \tilde{G}_{p}(p)
$$

The differentials $\phi_{* p}$ of all local diffeomorphisms which induce $A P S$ 's at $p$ form a subgroup $L_{p}(P)$ of the full linear group $L G\left(T_{p} M\right)$ of $T_{p} M$. Under the standard homomorphism $h: L G\left(T_{p} M\right) \rightarrow P G\left(D_{p} M\right)$ we have

$$
L_{p}(p) \rightarrow \tilde{G}_{p}(p) \text {. }
$$

We shall denote the center of $L G\left(T_{p} M\right)$, which is also the kernel of $h$, as $Z_{p}$ 。

By a dilation at $p$ we shall mean a local diffeomorphism $\phi$ of $M$ with fixed point $p$ such that $\phi_{* p} \in Z_{p}$, $\phi_{* p} \neq \mathrm{id}$.

Let $\phi$ be a local diffeomorphism leaving $p$ fixed. Take local coordinates in $M$ such that $x^{a}(p)=0 . \phi$ is then given by functions $\phi^{a}\left(x^{b}\right)$, and the expression for $\Phi_{* *}$ involves only

$$
\phi_{b}^{a}=\frac{\partial \phi^{a}}{\partial x^{b}} \text { and } \phi_{b c}^{a}=\frac{\partial^{2} \phi^{a}}{\partial x^{b} \partial x^{c}} .
$$

The direction field $\Xi$ of a path structure is given by the functions $v^{\alpha}\left(x^{b}, \xi^{\gamma}\right)$. If $\Psi$ is an $A P_{S}$ at $p$ induced by $\phi$, then $\Psi$ is completely determined by the numbers

$$
\Psi_{b}^{a}=\phi_{b}^{a}(0) \text { and } \Psi_{b c}^{a}=\phi_{b c}^{a}(0),
$$

and condition (5) is expressed (in one chart) by

$2 \xi^{a} \Psi_{a}^{[n}\left(\Psi_{b}^{\gamma]} v^{\sigma}\left(\xi^{\beta}\right)+\Psi_{b c}^{\gamma]} \xi^{b} \xi^{c}\right)=\left(\Psi_{b}^{n} \xi^{b}\right)^{3} v^{\gamma}\left(\frac{\Psi_{b}^{\beta} \xi^{b}}{\Psi_{b}^{n} \xi^{b}}\right)$

where we have written $v^{6}\left(\xi^{\beta}\right)$ instead of $v^{6}\left(0, \xi^{\beta}\right) . \phi$ is a dilatation at $p$ iff $\Psi_{b}^{a}=f \delta_{b}^{a}$ with $f \neq 0,1$.

Suppose $t \rightarrow \Psi(t)$ is a one-parameter subgroup of $G_{p}(P)$. With respect to local coordinates we can represent it by smooth functions $\Psi_{b}^{a}(t), \Psi_{b c}^{a}(t)$ satisfying $\Psi_{b}^{a}(0)=\delta_{b}^{a}$, $\Psi_{b c}^{a}(0)=0$. Taking derivatives at $t=0$ we obtain parameters $\psi_{b}^{a}=\Psi_{b}^{a^{\prime}}(0), \psi_{b c}^{a}=\Psi_{b c}^{a^{\prime}}(0)$ describing an element of the Lie algebra of $G_{p}(P)$. Applying this to Eq. (8) we obtain the infinitesimal version of the invariance condition,

$$
\begin{gathered}
\left.v_{{ }_{\beta} \beta}^{\gamma}\left(\xi^{\alpha}\right) \psi_{b}^{[\beta} \xi^{n 1} \xi^{b}+v^{\beta}\left(\xi^{\alpha}\right)\left(\psi_{b}^{n} \xi^{b} \delta_{\beta}^{\gamma}+\psi_{\beta}^{[n} \xi^{\gamma}\right]\right) \\
=\psi_{b c}^{[\gamma} \delta_{d}^{n 1} \xi^{b} \xi^{c} \xi^{d} .
\end{gathered}
$$

We now return to the discussion of path structures. A geodesic path structure $P_{\Gamma}$ admits at each point $p$ a group $G_{p}\left(p_{\Gamma}\right)$ of approximate symmetries whose image $G_{p}\left(P_{\Gamma}\right)$ [according to (6)] is the full group $P G\left(D_{p} M\right)$; also $L_{p}\left(P_{\Gamma}\right)=L G\left(T_{p} M\right)$. In particular $P_{\Gamma}$ admits everywhere approximate symmetries induced by dilatations. We shall now prove two theorems showing that the existence of some approximate symmetrices suffices, in turn, to characterize path structures as geodesic ones.

Theorem 2: A $C^{1}$ path structure $P$ is geodesic if and only if it admits at each point an approximate symmetry which is induced by a dilatation.

Proof: The necessity has been pointed out already. So, assume that $\phi$ is a dilatation at $p$ which induces an approximate $P$-symmetry. We then have, in local coordinates, Eq. (8) with $\Psi_{b}^{a}=f \delta_{b}^{a}, f \neq 0,1$. Consequently $v^{\gamma}$ is a polynomial in $\xi^{\beta}$. Since this holds at any point $p$, it follows from Theorem 1 that $P$ is geodesic.

This theorem can be understood intuitively: For $f>0$, a dilatation "stretches" or "compresses" $M$ radially away from or towards $p$. A path mapped into itself under this operation must be "infinitesimally straight." A similar idea applies if $f<0$. The characterization of geodesic path structures given in Theorem 2 is closely related to Weyl's elementary method to introduce a linear connection via locally geodesic coordinates. ${ }^{7}$

Theorem 3: A $C^{\omega}$ path structure $P$ is geodesic if and only if it admits, at each point $p \in M$, a group $G_{p}(P)$ of approximate symmetries which induces a transitive action in the set of projective bases of $D_{p} M$.

Proof: Again, the necessity has been established already. Let, then, $G_{p}(P)$ induce a transitive action in the set of bases of $D_{p} M$. Then $\widetilde{G}_{p}(P)=P G\left(D_{p} M\right)$. Relation (7) then shows that the corresponding group $L_{p}(P)$ satis fies $L_{p}(P) / L_{p}(P) \cap Z_{p}=P G\left(D_{p} M\right)$ whence $\operatorname{dim} L_{p}(P) \geqslant n^{2}-1$. Introducing again local coordinates we infer that the functions $v^{\gamma}$ describing $P$ satisfy a system of equations (9) not only for one system of parameters $\left(\psi_{b}^{a}, \psi_{b c}^{a}\right)$, but for a whole family of such systems containing $n^{2}-1$ linearly independent matrices $\psi_{b}^{a}$ [which represent elements of the Lie algebra of $\left.L_{p}(\dot{P})\right]$. Since $P$ is analytic the functions $v^{\gamma}\left(\xi^{\beta}\right)$ can be represented by power series. If these are inserted into (9) there results an infinite system of linear homogeneous equations in the unknowns $\psi_{b}^{a}$, the coefficients of which contain the expansion coefficients of $v^{\gamma}$ of degrees 4, 5, etc. (The lower degree terms appear in equations involving also the $\psi_{b c}^{a}$; these equations need not be considered.) The fact that this system admits $n^{2}-1$ linearly independent solutions $\left(\psi_{b}^{a}\right)$ implies that all the expansion coefficients of $v^{\gamma}$ of degree larger than 3 vanish, hence $v^{\gamma}$ is a polynomial, so that Theorem 1 gives the desired result. We give the laborious proof of the last part of this argument only for the case $n=2$. In this case $v=\sum_{0}^{\infty} \alpha_{i} \xi^{i}$ and (9) leads to $\alpha_{l+1}(l+1) \psi_{1}^{2}+\alpha_{l}\left[(2-l) \psi_{1}^{1}+(l-1) \psi_{2}^{2}\right]+\alpha_{l-1}(4-l) \psi_{2}^{1}=0$.

for $l \geqslant 4$. The coefficient matrix $A$ of this linear homogeneous system for $\psi=\left(\psi_{1}^{2}, \psi_{1}^{1}, \psi_{2}^{2}, \psi_{2}^{1}\right)$ reads

$$
A=\left[\begin{array}{cccc}
5 \alpha_{5} & -2 \alpha_{4} & 3 \alpha_{4} & 0 \\
6 \alpha_{6} & -3 \alpha_{5} & 4 \alpha_{5} & -\alpha_{4} \\
7 \alpha_{7} & -4 \alpha_{6} & 5 \alpha_{6} & -2 \alpha \\
8 \alpha_{8} & -5 \alpha_{7} & 6 \alpha_{7} & -3 \alpha_{6} \\
\vdots & \vdots & \vdots & \vdots
\end{array}\right]
$$

Since the space of solutions is at least three-dimensional, every two-dimensional subdeterminant of $A$ vanishes. This implies $A=0$, i.e., $v^{r}$ is a polynomial. For $n>2$ the argument is similar,

If $n=4$ and $M$ is interpreted as spacetime, we obtain 
the physical interpretation of Theorem 3 given in the Introduction.

We conjecture that Theorem 3 can be generalized to PS's of lower differentiability class, even to class $C^{2}$, by exploiting (9) or (8) more effectively.

\section{NONGEODESIC PATH STRUCTURES}

Although nongeodesic path structures have lower symmetry than geodesic path structures, they are not altogether physically uninteresting. To show the existence of such structures, consider the example in two dimensions given by

$$
v(\xi)=\frac{1-\xi^{5}}{1+\xi^{2}} \text { and } \vec{v}(\bar{\xi})=\frac{1-\bar{\xi}^{5}}{1+\bar{\xi}^{2}}
$$

which is clearly analytic. Parametrized curves representing the paths can be obtained as solutions of the differential equations

$$
\ddot{x}=\dot{y}^{4} /\left(\dot{x}^{2}+\dot{y}^{2}\right), \quad \ddot{y}=\dot{x}^{4} /\left(\dot{x}^{2}+\dot{y}^{2}\right), \quad(\dot{x}, \dot{y}) \neq(0,0) .
$$

(The corresponding spray on $T \mathbb{R}^{2}$ is of class $C^{1}$, but not $C^{2}$.) Another example, although only of class $C^{1}$, which is easily integrable, is

$$
v(\xi)=\xi^{4 / 3}, \quad \bar{v}(\bar{\xi})=-\bar{\xi}^{5 / 3} .
$$

Integral curves through $(0,0)$ are given by

$$
y=\frac{27}{2}\left[(x+b)^{-2}-b^{-2}\right], \quad b \neq 0
$$

plus the two coordinate axes.

To obtain an important physical example we slightly generalize the concept of a path structure.

Let $E$ be an open submanifold of $D M$ such that $\pi_{D}(E)$ $=M$. An $E$ path structure $P_{E}$ with domain $E$ is a set of paths in $M$ such that (a) through each point $p \in M$ and each direction $\xi_{p} \in E_{p}=E \cap \pi_{0}^{-1}(p)$ there passes exactly one path of the set, and (b) the lift of each path of $P_{E}$ into $D M$ is contained in $E$. An $E$ path structure will be called geodesic if there exists a connection $\Gamma$ on $M$ such that each path of $P_{E}$ can be represented by a geodesic of $\Gamma$.

Examples of $E$ path structures are collections of paths which are timelike with respect to a Lorentzian conformal structure of $M$. In this case $E$ is the set of timelike directions in $D M$.

We shall now consider a "timelike", nongeodesic path structure of physical importance. Let $\left(\mathbb{R}^{4}, \eta_{a b}\right)$ be the flat Minkowskian spacetime of special relativity, taken as time oriented, and let $F_{a b}\left(=-F_{b a}\right)$ be a 2 -form field on $\mathbb{R}^{4}$ interpreted as an electromagnetic field. Then the world lines of particles with specific charge 1 (say) are determined by

$$
\dot{x}^{a}=F_{b}^{a} \dot{x}^{b}, \quad \eta_{a b} \dot{x}^{a} \dot{x}^{b}=-1, \quad \dot{x}^{4}>0,
$$

where

$$
F_{b}^{a}=\eta^{a c} F_{c b} \text {. }
$$

Introducing direction coordinates $\xi^{\alpha}=\dot{x}^{\alpha} / \dot{x}^{4}(\alpha=1,2,3)$ as in Sec. 1 we see that timelike directions obey

$$
\left(\xi^{1}\right)^{2}+\left(\xi^{2}\right)^{2}+\left(\xi^{2}\right)^{2}<1
$$

and that the direction field corresponding to $(10)$ is given by $^{8}$

$v^{\alpha}\left(\xi^{\beta}\right)=\left(F^{\alpha}{ }_{4}-F^{\alpha}{ }_{B} \xi^{\beta}-F^{4}{ }_{\beta} \xi^{\beta} \xi^{\alpha}\right)\left[1-\left(\xi^{1}\right)^{2}-\left(\xi^{2}\right)^{2}-\left(\xi^{3}\right)^{2}\right]^{1 / 2}$.

Theorem 1 implies that the spacetime paths of charged particles with a fixed specific charge form a nongeodesic, timelike path structure. This examples can easily be generalized to an arbitrary Lorentzian manifold.

${ }^{1} \mathrm{H}$. Weyl, Nachr. Ges. Wiss. Göttingen 99 (1921), Mathematische Analyse des Raumproblens (Springer, Berin, 1923); J. Ehlers, F.A.E. Pirani, and A. Schild, "The Geometry of Free Fall and Light Propagation," in L. O'Raifeartaigh, Ed., General Relativity (Clarendon, Oxford, 1972), p. 63; M. Castagnino, J. Math. Phys. 12, 2203 (1971); F.A.E. Pirani and A. Schild, "Conformal Geometry and the Interpretation of the Weyl Tensor," in B. Hoffman, Ed., Perspectives in Geometry and Relativity (Indiana U.P., Bloomington, 1966), p. 291; J. Ehlers and A. Schild,

Commun. Math. Phys. 32, 119 (1973); F.A.E. Pirani, "Building Spacetime from Light Rays and Free Particles," in Symposia Mathematica Vol. XII (Academic, London, 1973), p. 67; J. Ehlers, "Survey of General Relativity Theory," in W. Israel, Ed., Relativity, Astrophysics and Cosmology (Reidel, Dordrecht, 1973), p. 1; N. M.J. Woodhouse, J. Math. Phys, 14, 495 (1973).

'See, e.g., E.H. Kronheimer and R. Penrose, Proc. Cambridge Philos. Soc. 63, 481 (1967); B. Carter, Gen. Rel. Grav. 1, 349 (1971); S.W. Hawking and G. F.R. Ellis, Large Scale Structure of Space Time (Cambridge U. 1'. Cambridge, 1973).

${ }^{3}$ S. Lang, Introduction to Differentiable Manifolds (Interscience, New York, 1962).

${ }^{4}$ "To avo id confusion we emphasize that the terms "curve" and "path" are not generally used in the sense described here. It is essential, however, to distingu ish between these concepts, and we shall adhere consistently to the terminology proposed in the text.

${ }^{5}$ See, e.g., D. Laugwitz, Differentialgeometrie (Teubner, Stuttgart, 1960), Sec. 15.3.

"In contrast to an "infinitesimal" transformation which, intuitively, is a map close to the identity, an "approximate" symmetry is "finite," but preserves the figure of interesthere $P_{p}$-only "infinitesimally."

${ }^{7} \mathrm{H}$. Weyl, Raum, Zeit, Materie (Springer, Berlin, 192:3), 5 th ed.

${ }^{8} \mathrm{Clearly}$ this direction field can be continuously extended to the set of null directions on which $v^{\alpha}$ vanishes. Hence, the limiting paths are represented by null geodesics. 